hard to the touch and they paled on pressure. They varied in size from a pin's head to a split pea. During the next few days the rash continued to extend, and in a couple of days after the first visit opalescence appeared in some of the papules and others displayed slight vesication, the vesicles being minute and containing a puriform secretion. A certain degree of general swelling was present for a day or two over the area of the eruption, but it rapidly subsided. Papules also appeared on the backs of the hands and fingers and fresh nodes appeared on the shins from day to day for the first week, all gradually passing through the usual succession of chromatic changes. The temperature remained slightly above $100^{\circ}$ for six days, with the exception of Jan. 3rd, 1901, when it sank to $99.5^{\circ}$. On the eighth day it was $99.8^{\circ}$ and it did not rise above this subsequently. The eruption gradually faded from both face and legs, leaving slight pigmentation behind. This pigmentation was visible on the face as late as the 20th-i.e., for about three weeks-but had disappeared by Jan. 25 th.

From the above history it will be evident that the nature of the lesione was unmistukeable. The slight rise of temperature, the local appearances, their symmetrical arrangement and their distribution, were all characteristic of erythema multiforme, while the condition of the shins was absolutely typical of erythema nodosum. The case, to my mind, is a very definite demonstration of the identity of erythema nodosum with erythema multiforme. The family history was remarkable. The patient's mother had similar " lumps on the legs" before marriage; his maternal grandmother had had rheumatic fever; an elder sister died at the age of 16 years from rheumatic endocarditis with muitiple joint affections; a younger sister was under my care last year with rheumatic fever, and had endocarditis and subsequent signs of mitral stenosis; another sister had had frequent attacks of acute tonsillitis (from which the patient himself has often suffered); and a maternal uncle and three of his children had had rheumatic fever. In contrast to this the patient's father is healthy and bas no rheumatic personal or family history. Thus additional support is given, if it be needed, to the view that erythema multiforme is but one of the expressions of rheumatism.

Torpoint, Devonport.

\section{NOTE ON THE TREATMENT OF OTORRHCEA.}

\section{By Dafid William Aitken, M.B., C.M. Edin.}

As the method about to be described has proved successful in cases where the alternative is antral perforation it seems well to call the attention of the profession to it. Although it is quite prompt in its effects upon acute otorrhœa its benefits are greatest in old-standing cases where the mastoid has become infected. Such cases have lasted for years there is an abominable discharge, probably with polypoid growths, and if the more threatening head symptoms are not present there are the impaired health, the atonic dyspepsia, the anæmia, and the loss of flesh to denote the dire effects upon the constitution which follow absorption.

The appliances required are a probe, some antiseptic lotion, and some absorbent cotton. The best probe for the purpose which I have seen is that supplied by Messrs. Hockin and Wilson. It has at the end two spiral teeth which, while they hold the wadding firmly, permit of its easy removal by rotating the stem counter clock-wise. The first step is to pour into the ear some of the lotion. Then take as large a plug of wadding as is deemed sufficient when screwed upon the probe to easily fit the meatus. It is now possible to make the probe and ear canal a suction syringe. The plug of wadding which forms the piston is gently pushed in and then withdrawn. If it is found to be either too large or too small another can be at once substituted which acts both easily and also fits close enough to force some of the fluid before it. This flaid reaches both the attic and also the mastoid recesses. At any rate, on the first withdrawal sufficient vacuum is produced to allow the lotion to enter the accessory cavities. It will surprise anyone who has not carried out this procedure to note how much discharge and débris are brought to the surface even after syringing and swabbing have been efficiently performed. After several repetitions of the mancuvre, the head each time being turned to the opposite side to permit of emptying the meatus, the lotion will well up clean. Now one can get any medicament to the clean surfaces. Begin with chinosol, iodoform, or amyloform in alcohol which, in my experience, is best in the absolute state. It is practically painless in almost all cases and in the exceptions the smarting is but momentary. Its advantages are : (1) it acts promptly upon the polypoid growths; (2) it is a most satisfactory antiseptic; and (3) as it evaporates it leaves a dry surface. This is most important. Many of the cases which progress so unsatisfactorily are those of patients who get a " powder to put in water to syringe the ear with." While the ear is never clean it is kept constantly in a sodden state. When the solution has been poured into the ear the process with the "piston-rod" is repeated several times. Thus the fluid is forced into all the recesses. That this is so is seen by the prompt improvement both in the local condition and also in the constitutional state. Of course, discretion is used as to the nature of the drug selected in the progress of the cure, according to the requirements-stimulant, astringent, sc.- - of the case. It is unnecessary to select examples. Suffice it to say that many cases have been treated and that in some the patients have probably been saved from the somewhat serious operation of trephining the mastoid.

It is my belief that this plan, if properly carried out, would greatly diminish the number of antral perforations. In fact, it is as an alternative to that procedure that my device has been matured.

Packington-street, $\mathbf{N}$.

\section{BARIUM WATER IN WEAKNESS OF THE HEART.}

BY W. BlaCK JoNes, M.D., B.S. LOND., D.P.H., PHYSICLAN TO THE LLANGAMMARCH WELLS BPA.

THE following case, which illustrates others of a similar nature treated at the Llangammarch Wells Spa, may be found to be of interest.

A man, aged 53 years, consulted me for weakness of the heart. He had had influenza seven years previously and since then had suffered from dyspncea on exertion. He had been losing flesh of late. He was a very thin man, weighing only seven stones. The apex beat of the heart was in the nipple line and the cardiac dulness was increased to a corresponding extent. At the apex a soft systolic murmur was beard which became louder as the right base was approached; over the pulmonary area the second sound was accentnated. The pulse was thin and wiry and the frequency varied from 90 to 100 per minute. Nothing abnormal was observed in the other organs; the urine was unaffected. The patient was advised to drink the Llangammarch barium water, to have a course of barium baths, and to take outdoor exercise in moderation. He soon began to improve and after three weeks' treatment he left, feeling considerably better. After an absence of six weeks he reported that be had made greater progress; the pulse was slower, he had practically lost the dyspncea, and he was able to walk up hill with comparative ease. He was also gaining in weight and was feeling better than he had for years.

Similar to the above case are many in which the heart has been damaged after attacks of influenza. They all receive considerable benefit from the treatment at Llangammarch and often gain considerably in strength after their return home.

Llangammarch Wells, Breconshire.

Society for Relief of Widows and Orphans OF MEdical MEN.-A quarterly court of the directors of this society was held on April 10th, Mr. Christopher Heath, the President, being in the chair. Ten new members were elected and the deaths of three were reported. The death of a widow was announced. Fresh applications for grants were read from two widows and three orpbans and grants amounting to $£ 96$ per annum were made and a grant of $£ 25$ for the self-maintenance of an orphan was given. Applications for continuation of grants were read from 50 widows, 14 orphans. and six on the Copeland Fund, and it was resolved that $£ 1218$ be given at the July court. The following were recommended for election at the annual general meeting to fill vacancies in the list of officers for 1801-2-viz.: Dr. Blandford as treasurer, and Mr. Sleeman, Mr. Elwin, Sir Hermann Weber, Mr. Stowers, Dr. Nix, and Dr. Palmer as directors. It was decided to hold the annual general meeting on May 22 nd at 5 P.M. 